DAMTP/R-93/5

CTP-TAMU-23/94

\title{
Macroscopic superstrings as interpolating solitons
}

\author{
M.J. Duff*, G.W. Gibbons** and P.K. Townsend** \\ *: Dept. of Physics, Texas A\& M Univ., \\ College Station, Texas, U.S.A. \\ **:DAMTP, Univ. of Cambridge, \\ Silver St., Cambridge, U.K.
}

\begin{abstract}
It is known that, in string sigma-model metric, the 'extreme' fivebrane solution of $\mathrm{D}=10$ supergravity interpolates between $\mathrm{D}=10$ Minkowski spacetime and a supersymmetric $S^{3}$ compactification to a linear dilaton vacuum. We show here that, in fivebrane sigma-model metric, the extreme string solution of $\mathrm{D}=10$ supergravity interpolates between Minkowski spacetime and a hitherto unknown supersymmetric $S^{7}$ compactification of $d=10$ supergravity to a three-dimensional anti-de Sitter generalization of the linear dilaton vacuum, which may be invariantly characterized in terms of conformal Killing vectors. The dilaton field diverges near the string core but this divergence may be eliminated by re-interpreting the string solution as the extreme membrane solution of 11-dimensional supergravity. We show that the latter has an analytic extension through a regular degenerate event horizon to an interior region containing a curvature singularity. We obtain analogous results for other extended object solutions of supergravity theories.
\end{abstract}




\section{Vacuum interpolation via macroscopic superstrings}

The action for the bosonic sector of $N=1 D=10$ supergravity, which can be viewed as (part of) the field theory limit of the heterotic string, can be written as

$$
S=\frac{1}{2 \kappa^{2}} \int d^{10} x \sqrt{-g} e^{-2 \phi}\left[R+4(\partial \phi)^{2}-\frac{1}{12} F_{3}^{2}\right]
$$

where $F_{3}$ is the three-form field-strength for a two-form potential, and $\phi$ is the dilaton field. There is an intrinsic ambiguity in the spacetime metric in theories with a dilaton because a new, although conformally equivalent, metric may be obtained by rescaling the old one by any positive function of the dilaton, e.g. by a power of $e^{\phi}$. A choice of metric in this conformal equivalence class corresponds to a choice of positive function of $\phi$ multiplying the Einstein term in the action. The choice of $e^{-2 \phi}$, as in (1.1), corresponds to the string sigma-model metric since this is the metric that couples to the worldsheet of the string. Non-singular spacetimes in this metric that solve the field equations may be regarded as approximate solutions of string theory. For the purposes of this paper we shall say that a spacetime with metric $g_{\mu \nu}$, dilaton $\phi$ and $q$-form field strength $F_{q}$ is non-singular if

(i) $g_{\mu \nu}$ is geodesically complete

(ii) $\phi$ is everywhere finite and smooth

(iii) $F_{q}$ has everywhere finite and smooth components in a coordinate system in which $g_{\mu \nu}$ has finite and smooth components.

An example is the extreme 'elementary' fivebrane solution [1]

$$
\begin{aligned}
d s^{2} & =-d t^{2}+d \mathbf{x} \cdot d \mathbf{x}+\left[1-\left(\frac{a}{r}\right)^{2}\right]^{-2} d r^{2}+r^{2} d \Omega_{3}^{2} \\
e^{-2 \phi} & =\left[1-\left(\frac{a}{r}\right)^{2}\right] \\
F_{3} & =2 a^{2} \varepsilon_{3}
\end{aligned}
$$

where $d \mathbf{x} \cdot d \mathbf{x}$ is the Euclidean 5-metric, $d \Omega_{3}^{2}$ the standard metric on the unit 3-sphere and $\varepsilon_{3}$ its volume 3 -form, and $a$ is a constant. Evidence for the interpretation of this solution as an 'extended soliton' is provided by a recent demonstration [2] that it interpolates between two supersymmetric vacua of $N=1 d=10$ supergravity, one being $\mathrm{d}=10$ Minkowski space and the other a previously unknown $S^{3}$ compactification of $d=10$ supergravity to seven-dimensional Minkowski spacetime with a linear dilaton.

A class of string-like solutions of the field equations of the action (1.1) was found in [3]. In the 'extreme' limit of interest to us here, and expressed in terms of the seven form 
$F_{7} \equiv e^{-2 \phi} \star F_{3}$, where $\star$ indicates the Hodge dual, the solution takes the form

$$
\begin{aligned}
d s^{2} & =\left[1-\left(\frac{a}{r}\right)^{6}\right]\left[-d t^{2}+d \sigma^{2}\right]+\left[1-\left(\frac{a}{r}\right)^{6}\right]^{-\frac{5}{3}} d r^{2}+r^{2}\left[1-\left(\frac{a}{r}\right)^{6}\right]^{\frac{1}{3}} d \Omega_{7}^{2} \\
e^{-2 \phi} & =\left[1-\left(\frac{a}{r}\right)^{6}\right]^{-1} \\
F_{7} & =6 a^{6} \varepsilon_{7}
\end{aligned}
$$

where $d \Omega_{7}^{2}$ is the standard metric on the unit 7 -sphere and $\varepsilon_{7}$ its volume 7 -form. Unlike the spacetime metric of the fivebrane solution, the metric (1.2) is singular as $r \rightarrow a$. But this is the string sigma-model metric; the fact that a string in $D=10$ is dual to a fivebrane suggests that we instead consider the metric coupling to the six-dimensional worldvolume of a fivebrane, i.e. the fivebrane sigma-model metric, which is the conformally rescaled metric $[4]$

$$
d \tilde{s}^{2} \equiv e^{-\frac{2}{3} \phi} d s^{2}=\left[1-\left(\frac{a}{r}\right)^{6}\right]^{\frac{2}{3}}\left[-d t^{2}+d \sigma^{2}\right]+\left[1-\left(\frac{a}{r}\right)^{6}\right]^{-2} d r^{2}+r^{2} d \Omega_{7}^{2}
$$

This rescaled metric is still singular on the horizon at $r=a$ but now the singularity is merely due to an inappropriate choice of coordinates. As a first step towards this result, proved in section 3, we now investigate the asymptotic form of the metric near $r=a$. As in [2] we define

$$
\lambda=\frac{6(r-a)}{a}
$$

to get

$$
d \tilde{s}^{2}=\left\{\lambda^{\frac{2}{3}}\left[-d t^{2}+d \sigma^{2}\right]+\frac{a^{2}}{36} \lambda^{-2} d \lambda^{2}+a^{2} d \Omega_{7}^{2}\right\}(1+O(\lambda))
$$

Neglecting the $O(\lambda)$ terms, and defining

$$
\rho=\frac{a}{6} \ln \lambda
$$

we find that

$$
d \tilde{s}^{2} \sim e^{\frac{4 \rho}{a}}\left(-d t^{2}+d \sigma^{2}\right)+d \rho^{2}+a^{2} d \Omega_{7}^{2}
$$

which is the standard metric on the product of $S^{7}$ with three-dimensional anti-de Sitter space, $a d S_{3}$.

The asymptotic form of the dilaton (near $r=a$ ) in the new coordinates is

$$
\phi \sim 3 \frac{\rho}{a}
$$

i.e. a linear dilaton. Clearly, the dilaton is linear only for the special choice of coordinates used here and since the spacetime factor of the asymptotic metric is not Minkowski (as it was for the fivebrane solution) but rather $a d S_{3}$, the geometrical significance of linearity is unclear from the above result. We shall return to this point below, but it already clear 
from (1.9) that the dilaton diverges on the horizon at $r=a$, so that the extreme string solution of (1.3), as against just the metric, is singular there.

If the action (1.1) is now rewritten in terms of the new, fivebrane sigma-model, metric, and with $F_{7}$ in place of $F_{3}$, it becomes

$$
S \rightarrow \tilde{S}=\frac{1}{2 \kappa^{2}} \int d^{10} x \sqrt{-g} e^{\frac{2}{3} \phi}\left[R-\frac{1}{2.7 !} F_{7}^{2}\right]
$$

where $F_{7}$ may now be interpreted as the field strength of a six-form potential satisfying the Bianchi-identity $d F_{7} \equiv 0$. We have just shown that the extreme string solution of the field equations of this action interpolates between $D=10$ Minkowski spacetime and $a d S_{3} \times S^{7}$ near the core, with a 'linear' dilaton that diverges at $r=a$. In this respect the string solution in fivebrane sigma-model metric is similar to the fivebrane solution in string sigma-model metric, as string/fivebrane duality would suggest. We verify below that the $S^{7}$ compactification of $\mathrm{D}=10$ supergravity implied by this analysis indeed exists, incidentally elucidating the geometrical significance of 'linear' in the adS context.

In other respects, however, the string solution in fivebrane sigma-model metric is quite different from the fivebrane solution in string sigma-model metric. In particular, the string solution has an event horizon at which the dilaton diverges, so it fails to be a non-singular solution of $D=10$ supergravity, in the sense of this paper*. However, we shall show that the divergence of the dilaton at the horizon may be eliminated by re-interpreting the extreme string solution as the extreme membrane solution [5] of $D=11$ supergravity. The latter solution still has an event horizon but now one can continue the solution analytically through it to a region containing a singularity at $r=0$. So even in $D=11$ the solution is not completely non-singular but is rather more analogous to the four-dimensional extreme Reissner-Nordstrom solution in which a singularity is hidden behind a degenerate event horizon.

\section{The $S^{7}$ compactification}

Let $\left\{x^{M} ; M=0,1, \ldots, 9\right\}$ be coordinates for the ten-dimensional spacetime, and define

$$
\Phi=e^{\frac{2}{3} \phi} .
$$

The field equations of (1.10) can now be written as

$$
R_{M N}=\Phi^{-1} D_{M} D_{N} \Phi+\frac{1}{2.6 !} F_{M P_{1} \ldots P_{6}} F_{N} P_{1} \ldots P_{6}-\frac{1}{3.7 !} g_{M N} F_{7}^{2}
$$

* Whether this is a 'physical' singularity is a more subtle question. In [4] it was shown using a test probe/source approach that if the test probe and source are both strings or both fivebranes, the probe falls into $\mathrm{r}=\mathrm{a}$ in a finite proper time. If one is a string and the other a fivebrane, however, it takes an infinite proper time. So by these different criteria, the singularity structure is symmetrical between strings and fivebranes. 


$$
\begin{gathered}
\partial_{M}\left(\sqrt{-g} \Phi F^{M N_{1} \ldots N_{6}}\right)=0 \\
\square \Phi=\frac{1}{3.7 !} F_{7}^{2} \Phi
\end{gathered}
$$

We now split the coordinates $\left\{x^{M}\right\}$ into $\left\{x^{\mu}, y^{m}\right\}$ with $\mu=0,1,2$ and $m=1, \ldots, 7$, and seek product metrics of the form

$$
g_{\mu \nu}=g_{\mu \nu}(x) \quad g_{m n}=g_{m n}(y)
$$

with $g_{\mu n}=0$. In this case, $\sqrt{-g}=e_{3}(x) e_{7}(y)$ where $e_{3}$ and $e_{7}$ are the scalar density volume factors for the three and seven-dimensional spaces, respectively. It follows that $(2.2 \mathrm{~b})$ is now solved by setting

$$
F^{m_{1} \ldots m_{7}}=3 m\left(e_{7}\right)^{-1} \varepsilon^{m_{1} \ldots m_{7}}
$$

for some constant $m$, with all other components of $F_{7}$ vanishing, whereupon $(2.2 \mathrm{c})$ becomes

$$
\left(\square-3 m^{2}\right) \Phi=0
$$

We now suppose that $\Phi=\Phi(x)$, and further that

$$
D_{\mu} \partial_{\nu} \Phi=\frac{1}{3} g_{\mu \nu} \square \Phi
$$

or, equivalently, in view of $(2.5)$,

$$
D_{\mu} \partial_{\nu} \Phi=m^{2} g_{\mu \nu} \Phi
$$

which implies (2.5) and therefore supercedes it. Given (2.7), (2.2a) reduces to the two equations

$$
R_{\mu \nu}=-2 m^{2} g_{\mu \nu} \quad R_{m n}=\frac{3}{2} m^{2} g_{m n}
$$

with $R_{m \nu}=0$. These equations are solved by the standard invariant metrics on $a d S_{3}$ and $S^{7}$ respectively. It remains to solve $(2.7)$. We can choose coordinates $(t, \sigma, \rho)$ on $a d S_{3}$ such that the $a d S_{3}$ metric is

$$
d s^{2}=e^{2 m \rho}\left(-d t^{2}+d \sigma^{2}\right)+d \rho^{2}
$$

In these coordinates it is straightforward to verify that

$$
\phi=\frac{3 m}{2} \rho
$$

solves (2.7). We have therefore found an $S^{7}$ compactification of $D=10$ supergravity to $a d S_{3}$ with a linear dilaton. Setting $m=2 / a$ we recover (1.8) and (1.9), found previously as an asymptotic limit of the extreme string solution.

Observe now that (2.6) implies that $k=g^{\mu \nu} \partial \mu \Phi \partial_{\nu}$ is a conformal Killing vector of $a d S_{3}$. We have shown above that an eigenfunction of the Dalembertian on $a d S_{3}$ with 
eigenvalue $3 m^{2}$ is the potential for a conformal Killing vector of $a d S_{3}$. A similar observation was made previously in the context of an $S^{3}$ compactification of $D=10$ supergravity [6] to $a d S_{7}$. In fact, the $S^{7}$ compactification exhibited above is obtained from the solution found in [6] by the analytic continuation $m \rightarrow i m$. We now see that the linear dilaton can be characterized in a coordinate-free way as proportional to the logarithm of a conformal Killing potential.

\section{Eleven-dimensional interpretation}

The extreme string solution of $D=10$ supergravity theory has a (fivebrane sigmamodel) metric which is non-singular (in appropriate coordinates) on the horizon $r=a$. As for the extreme Reissner-Nordstrom solution of $D=4$ Einstein-Maxwell theory, this metric can be continued through the horizon to $r<a$, but it would seem, on the face of it, that the dilaton field cannot be extended through $r=a$ because (1.3) tells us that $\phi \rightarrow \infty$ as the horizon is approached and that it is complex for $r<a^{*}$. However, the field $\Phi$, defined in (2.1), is given by

$$
\Phi=\left[1-\left(\frac{a}{r}\right)\right]^{\frac{1}{3}},
$$

which certainly remains real and bounded as we pass through the horizon. Moreover, the dilaton $\phi$ appears in the action (1.10) through $\Phi$ only. It is known that 10-dimensional supergravity can be viewed as the dimensional reduction of 11-dimensional supergravity and this allows a re-interpretation of $\Phi^{2}$ as a component of an 11-metric. We shall show that the above macroscopic string solution is in fact non-singular at $r=a$ when interpreted as a solution of 11-dimensional supergravity.

The 11-dimensional solutions of interest are

$$
\begin{aligned}
d s_{11}^{2} & =\Phi^{2}\left(d x^{11}\right)^{2}+d \tilde{s}^{2} \\
\star F_{4} & =6 a^{6} \varepsilon_{7}
\end{aligned}
$$

where $d s_{11}^{2}$ and $F_{4}$ are the metric and 4 -form of 11-dimensional supergravity (and $\star$ is the Hodge dual in 11 dimensions) and $d \tilde{s}^{2}$ is the $\mathrm{D}=10$ fivebrane sigma-model metric. In eleven dimensions there is no dilaton field and no possibility of conformal rescaling to avoid singularities, so the question of whether the eleven-dimensional fields are singular reduces to whether the metric of (3.2) is singular.

We begin by examining the $S^{7}$ compactification discussed in section (2) from this new point of view. In this case $\Phi=e^{m \rho}$ so

$$
d s_{11}^{2}=e^{2 m \rho}\left(-d t^{2}+d \sigma^{2}+d x_{11}^{2}\right)+d \rho^{2}+4 m^{-2} d \Omega_{7}^{2} .
$$

Since the first four terms in (3.3) give the metric of $a d S_{4}$, it is clear that, at least locally, (3.3) is just the standard $a d S_{4} \times S^{7}$ vacuum of eleven-dimensional supergravity. The

* This problem does not arise for the fivebrane solution in string conformal gauge because the metric in that case has no horizon. 
ten-dimensional solution corresponding to (2.9) may be identified as the ten-dimensional hypersurface $x^{11}=0$. Since this hypersurface is invariant under the involution $x^{11} \rightarrow-x^{11}$, it has vanishing second fundamental form, i.e. it is a totally geodesic submanifold of the eleven-dimensional spacetime.

The global structure of the spacetime (3.3) is governed by the behaviour of its $a d S_{4}$ factor. The 'horospherical' coordinates $\left\{\rho, t, \sigma, x^{11}\right\}$ do not cover all of $a d S_{4}$ but, rather, just half of it; in fact, they cover $a d S_{4} / J$ where $J$ is the antipodal map. To see why this is so, it is convenient to regard $a d S_{4}$ as the quadric $Q$ in $R^{3,2}$ given by

$$
\left(X^{0}\right)^{2}+\left(X^{4}\right)^{2}-\left(X^{1}\right)^{2}-\left(X^{2}\right)^{2}-\left(X^{3}\right)^{2}=\frac{1}{m^{2}}
$$

One has

$$
\begin{aligned}
m\left(X^{4}-X^{3}\right) & =\Phi, \\
m X^{0} & =t \Phi, \\
m X^{1} & =\sigma \Phi, \\
m\left(X^{4}+X^{3}\right) & =\Phi^{-1}+\left(\sigma^{2}+\left(x^{11}\right)^{2}-t^{2}\right) \Phi,
\end{aligned}
$$

The hypersurfaces $\rho=$ const. in $a d S_{4}$ correspond to the intersection of the null hyperplanes, $N$, in $R^{3,2}$, defined by

$$
m\left(X^{4}-X^{3}\right)=e^{m \rho}=e^{\frac{2}{3} \phi}=\Phi,
$$

with the quadric $Q$. However, as long as $\rho$ is taken to be real (corresponding by (2.10) to real $\phi$ ) there is no way to cover that half of $a d S_{4}$ for which $X^{4}-X^{3}$ is negative. The boundary of the region for which $X^{4}-X^{3}$ is positive is a null hypersurface $\mathcal{H}$ in $a d S_{4}$ obtained by intersecting the the quadric $Q$ with the null hyperplane, $N_{0}$, passing through the origin, i.e. $X^{4}-X^{3}=0$. The Killing vectors $\frac{\partial}{\partial t}, \frac{\partial}{\partial \sigma}$ and $\frac{\partial}{\partial x^{11}}$ are, respectively, timelike, spacelike and spacelike everywhere on $a d S_{4}$ except on the null hypersurface $\mathcal{H}=Q \cap N_{0}$. On $\mathcal{H}$, which is a degenerate Killing horizon, and corresponds to the event horizon $r=a$ in the extreme string solution, these Killing vectors are null. Note that the null hyperplane $N_{0}$ divides $a d S_{3}$ into two isometric pieces, that for which $X^{4}>X^{3} \Leftrightarrow \Phi>0$ and that for which $X^{4}<X^{3} \Leftrightarrow \Phi<0$. These two halves are interchanged by the antipodal involution $J: X^{A} \rightarrow-X^{A} A=0,1,2,3,4$. Evidently, from the point of view of the geometry of ad $S_{4}$ there is no reason for $\phi$ to be real or finite, nor for $\Phi$ to be positive.

Note that on $a d S_{4}$ the horizon $\mathcal{H}$ has two connected components, $\mathcal{H}^{+}$and $\mathcal{H}^{-}$, corresponding to the two values of $X^{0}$ satisfying (3.4) with $\Phi=m\left(X^{4}-X^{3}\right)=0$. One component, $\mathcal{H}^{-}$, is the past horizon and one component, $\mathcal{H}^{+}$, is the future horizon. On the universal covering space $\widetilde{a d S}_{4}$ there are infinitely many such pairs. On the identified space $a d S_{4} / J$ the two components $\mathcal{H}^{+}$and $\mathcal{H}^{-}$are identified.

The above discussion for $a d S_{4}$ may readily be extended to the string metric (1.4). In this case $\Phi$ is given by (3.1) and the corresponding eleven-dimensional metric is

$$
d s_{11}^{2}=\left[1-\left(\frac{a}{r}\right)^{6}\right]^{\frac{2}{3}}\left[-d t^{2}+d \sigma^{2}+\left(d x^{11}\right)^{2}\right]+\left[1-\left(\frac{a}{r}\right)^{6}\right]^{-2} d r^{2}+r^{2} d \Omega_{7}^{2}
$$


Observe that this metric can be interpreted as that of a membrane rather than a string. In fact, it is just the membrane solution of [5] but written in terms of our Schwarzschild-type radial coordinate $r$ rather than the isotropic radial coordinate of [5], which we shall call $\hat{r}$; the relation between the two is $\hat{r}=\left(r^{6}-a^{6}\right)^{\frac{1}{6}}$. In [5] this solution was interpreted as the metric exterior to a singular membrane source located at $\hat{r}=0$, i.e. at the horizon. Fortunately one need not suppose the existence of such a source since the metric can be smoothly extended through $r=a$ by choice of a suitable set of coordinates, although, like the extreme Reissner-Nordstrom black hole, there is a singularity hidden behind the horizon, at $r=0$. One way of showing this is to follow closely the discussion for the $a d S_{4}$ case. There, the four coordinates $\left\{X^{0}, X^{1}, X^{2}, X^{3}\right\}$ (defined in terms of $\left\{\Phi, t, \sigma, x^{11}\right\}$ by eqs. (3.5)), cover the horizon $\mathcal{H}$, with $\Phi=e^{m \rho}$; the field $\Phi$ is a well-behaved function on $a d S_{4}$ and one may check that the metric written in terms of the coordinates $\left\{X^{0}, X^{1}, X^{2}, X^{3}\right\}$ is well-behaved in a neighbourhood of the horizon. In the case of the metric (3.7) one can still use $\left\{X^{0}, X^{1}, X^{2}, X^{3}\right\}$ defined in terms of $\left\{\Phi, t, \sigma, x^{11}\right\}$ by (3.5) but now one uses (3.1) to express this metric in terms of $\left\{X^{0}, X^{1}, X^{2}, X^{3}\right\}$. Thus we set

$$
r=a\left(1-\Phi^{3}\right)^{-\frac{1}{6}}
$$

and re-express $(3.7)$ in terms of $\left(\Phi, t, \sigma, x^{11}\right)$ to get

$$
\begin{aligned}
d s_{11}^{2}=\left\{\Phi ^ { 2 } \left[-d t^{2}\right.\right. & \left.\left.+d \sigma^{2}+\left(d x^{11}\right)^{2}\right]+4 a^{2} \Phi^{-2} d \Phi^{2}+a^{2} d \Omega_{7}^{2}\right\} \\
& +a^{2}\left[\left(1-\Phi^{3}\right)^{-\frac{1}{3}}-1\right]\left[4 \Phi^{-2} d \Phi^{2}+d \Omega_{7}^{2}\right] .
\end{aligned}
$$

That is,

$$
d s_{11}^{2}=d s_{11}^{2} \text { (asymptotic) }+a^{2}\left[\left(1-\Phi^{3}\right)^{-\frac{1}{3}}-1\right]\left[4 \Phi^{-2} d \Phi^{2}+d \Omega_{7}^{2}\right]
$$

where the first term on the right hand side is asymptotic metric as $r \rightarrow a$, i.e. the standard metric on $a d S_{4} \times S^{7}$. Since the function $\Phi=m\left(X^{4}-X^{3}\right)$ is an analytic function on $a d S_{4}$ the remaining terms in (3.10) extend analytically through the horizon $\mathcal{H}$, at $r=a$, to give an analytic metric up to the curvature singularity at $\Phi=-\infty$, i.e. $r=0$.

The local chart we have introduced contains two connected components of the horizon, $\mathcal{H}^{+}$and $\mathcal{H}^{-}$. If one continues the extension to obtain the maximal analytic simplyconnected extension, there will be, just as for $\widetilde{a d S}_{4}$, infinitely many pairs of such horizons. The Carter-Penrose diagram obtained by supressing the angular coordinates on $S^{7}$ and the two ignorable worldsheet coordinates $\sigma$ and $x^{11}$ resembles that of the extreme ReissnerNordstrom metric.

It follows from the above analysis, by simply setting $x^{11}=0$, that the $D=10$ metric of (1.4) can also be extended analytically through the horizon to an interior region with a singularity at $r=0$, so we have now established that the singularity of this metric at $r=a$ is merely a coordinate singularity. The higher-dimensional interpretation is required only to circumvent the singularity of the $D=10$ dilaton field on the horizon

\section{Generalization to super $p$-branes}


We have seen that the ten-dimensional macroscopic superstring interpolates between the flat ten-dimensional Minkowski vacuum and a compactification on $S^{7}$ to $a d S_{3}$ with a 'linear' dilaton, which is invariantly characterized in terms of a conformal Killing potential. We also saw that the problem of the divergence of the dilaton at the horizon could be circumvented by passing to a non-singular 11-dimensional spacetime, re-interpreting the solution as a membrane. We shall now investigate to what extent these features persist in a class of metrics representing macroscopic $p$-branes in a $D$-dimensional spacetime. We consider an action of the form

$$
S=\frac{1}{2 \kappa^{2}} \int d^{D} x \sqrt{-g}\left[R-\frac{1}{2}(\partial \phi)^{2}-\frac{1}{2(d+1) !} e^{-\alpha \phi} F_{d+1}^{2}\right]
$$

where $F_{d+1}$ is a field-strength for a d-form potential $A$, and $\alpha$ is a constant. This action is expressed in terms of a metric that we shall call the Einstein metric, since the Einstein term is the canonical one, without factors of $e^{\phi}$. The action in terms of the $(d-1)$-brane sigma-model metric, i.e. the metric coupling to the d-dimensional worldvolume of such an object, is obtained by the conformal rescaling

$$
g_{\mu \nu} \rightarrow e^{-\frac{\alpha}{d} \phi} g_{\mu \nu}
$$

which yields

$$
\begin{aligned}
S=\frac{1}{2 \kappa^{2}} \int d^{D} x \sqrt{-g} e^{-\frac{(D-2) \alpha}{2 d} \phi}[R- & \frac{1}{2}\left(1-\frac{\alpha^{2}(D-1)(D-2)}{2 d^{2}}\right)(\partial \phi)^{2} \\
& \left.-\frac{1}{2(d+1) !} F_{d+1}^{2}\right] .
\end{aligned}
$$

For $\alpha$ satisfying

$$
\alpha^{2}=4-\frac{2 d \tilde{d}}{(D-2)}
$$

extreme $(\tilde{d}-1)$-brane solutions of the equations of motion of (4.1) are given in [7], where

$$
\tilde{d}=D-d-2
$$

After the rescaling of (4.2) these solutions are

$$
\begin{aligned}
d s^{2} & =\Delta^{\frac{d-2}{d}}\left(-d t^{2}+d \mathbf{x} \cdot d \mathbf{x}\right)+\Delta^{-2} d r^{2}+r^{2} d \Omega_{d+1}^{2} \\
e^{-2 \phi} & =\Delta^{\alpha} \\
F_{d+1} & =d a^{d} \varepsilon_{d+1}
\end{aligned}
$$

where $d \mathbf{x} \cdot d \mathbf{x}$ is the Euclidean $(\tilde{d}-1)$-metric, and

$$
\Delta=1-\left(\frac{a}{r}\right)^{d}
$$


For $D=10$ these solutions include the fivebrane and string solutions previously discussed.

To determine the asymptotic form of the metric of (4.6) near $r=a$ we let

$$
r=a\left(1+\frac{\lambda}{d}\right)
$$

in which case

$$
d s^{2}=\left[\lambda^{\frac{d-2}{d}}\left(-d t^{2}+d \mathbf{x} \cdot d \mathbf{x}\right)+\left(\frac{a}{d}\right)^{2} \lambda^{-2} d \lambda^{2}+a^{2} d \Omega_{d+1}^{2}\right](1+O(\lambda)) .
$$

Neglecting the $O(\lambda)$ terms, as before, and defining the new coordinate $\rho$ by

$$
\lambda=e^{\frac{d}{a} \rho}
$$

we get

$$
\begin{aligned}
d s^{2} & \sim e^{\frac{(d-2)}{a} \rho}\left(-d t^{2}+d \mathbf{x} \cdot d \mathbf{x}\right)+d \rho^{2}+a^{2} d \Omega_{d+1}^{2} \\
\phi & \sim-\frac{d \alpha}{2 a} \rho \\
F_{d+1} & \sim d a^{d} \varepsilon_{d+1} .
\end{aligned}
$$

This agrees with (1.8) and (1.9) for the extreme string solution of $D=10$ supergravity discussed in section 1 , for which $\alpha=-1^{*}$

If $d=2$ (for which $\alpha \neq 0$ ), then (4.11) reduces to

$$
\begin{aligned}
d s^{2} & \sim\left(-d t^{2}+d \mathbf{x} \cdot d \mathbf{x}+d \rho^{2}\right)+a^{2} d \Omega_{d+1}^{2} \\
\phi & \sim-\frac{\alpha}{a} \rho \\
F_{3} & \sim 2 a^{2} \varepsilon_{3}
\end{aligned}
$$

which is $\left(\operatorname{Mink}_{\tilde{d}+1} \times S^{3}\right.$, with a linear dilaton vacuum. That is, in string sigma-model metric the dual $(\tilde{d}-1)$-brane interpolates between $D$-dimensional Minkowski spacetime and the product of $S^{3}$ with a $(\tilde{d}+1)$-dimensional Minkowski spacetime, generalizing the case of the $D=10$ fivebrane, which was noted in [2] to have this property. For these cases the asymptotic behaviour of the dilaton near the p-brane core can be invariantly characterized as linear in an ignorable coordinate associated with a space-translation Killing vector. Since there is no event horizon the $(\tilde{d}-1)$-brane solution is completely non-singular.

If $d \neq 2$ the asymptotic spacetime spacetime is $(a d S)_{\tilde{d}+1} \times S^{d+1}$, and there is an event horizon at $r=a$. In terms of the new coordinate

$$
z=-\frac{2 a}{(d-2)} e^{-\frac{(d-2)}{2 a} \rho}
$$

* And corrects a factor of 2 error in the result of [2] for the extreme fivebrane solution, for which $\alpha=1$; note that $\alpha \rightarrow-\alpha$ under duality. 
the asymptotic adS metric near the horizon is

$$
\left(\frac{2 a}{(d-2) z}\right)^{2}\left(-d t^{2}+d \mathbf{x} \cdot d \mathbf{x}+d z^{2}\right)
$$

Clearly, the vector

$$
k=\left(\frac{d-2}{2 a}\right) \frac{\partial}{\partial z}=\frac{\partial}{\partial \rho}\left(e^{\frac{(d-2)}{2 a} \rho}\right) \frac{\partial}{\partial \rho}
$$

is a conformal Killing vector with conformal Killing potential

$$
\Phi=e^{\frac{(d-2)}{2 a} \rho}=e^{-\frac{(d-2)}{d \alpha} \phi}
$$

that is

$$
k^{\mu}=g^{\mu \nu} \partial_{\nu} \Phi .
$$

This provides an invariant characterization of the 'linear dilaton' vacuum in the general $d \neq 2$ case.

The solutions just described for $d \neq 2$ cannot be considered as non-singular in $D$ dimensions because the dilaton is singular on the event horizon. We now investigate whether this problem can be circumvented by re-interpreting the solution as a $\tilde{d}$-brane in a $(D+1)$-dimensional spacetime, as was possible for the $D=10$ macroscopic superstring. From (4.3) we see that this is certainly possible if

$$
\alpha^{2}=\frac{2 d}{(D-1)(D-2)}
$$

because the absence of a $(\partial \phi)^{2}$ term allows an immediate interpretation in $(D+1)$ dimensions with $\Phi^{2}$ as the component of the metric in the extra dimension. This is compatible with the restriction on $\alpha$ assumed for the class of solutions being considered here, eq. (4.4), when $D$ satisfies

$$
D=\frac{(d-1)(d+2)}{(d-2)}=(d+3)+\frac{4}{(d-2)},
$$

which allows only $D=9$ and $D=10$. The $D=9$ case occurs for $d=4$, i.e. $\tilde{d}=5$, so this case represents a dilatonic $D=9$ membrane which, as we have now shown, can be reinterpreted as a $D=10$ threebrane, in fact as the selfdual threebrane of Horowitz and Strominger [8]. There are two $D=10$ cases. One occurs for $d=6$ and is just the macroscopic string solution already discussed. The other occurs for $d=3$, i.e. $\tilde{d}=5$, so this case represents a dilatonic $D=10$ fourbrane which we see can be interpreted as a $D=11$ fivebrane. In fact, it is Güven's fivebrane solution of $D=11$ supergravity [9].

In each of these cases the $(D+1)$-metric takes the form

$$
\begin{aligned}
d s^{2}= & {\left[1-\left(\frac{a}{r}\right)^{(D-p-2)}\right]^{\frac{1}{p+1}}\left[-d t^{2}+d \mathbf{x} \cdot d \mathbf{x}+\left(d x_{*}\right)^{2}\right] } \\
& +\left[1-\left(\frac{a}{r}\right)^{(D-p-2)}\right]^{-2}+r^{2} d \Omega_{d+1}^{2}
\end{aligned}
$$


where $p$ is the dimension of the extended object (now $\tilde{d}$ rather than $\tilde{d}-1$ ) and $x_{*}$ is the coordinate of the extra dimension of spacetime. For $d>1$ the only other way to re-interpret the action in $(D+1)$ dimensions is by interpreting the $d$-form potential as a mixed component of a $d+1$ form potential in $(D+1)$ dimensions and this has the effect of replacing $d$ by $\tilde{d}$ on the right hand side of (4.18) and (4.19), and hence allows the same values of $D$. The $(D+1)$-dimensional solutions are the same as above*.

The metrics (4.20) belong to a general class of $p$-brane metrics for arbitrary $p$ and general spacetime dimension, although only a few are solutions of supergravity theories. All have an event horizon and using the techniques described earlier for the macroscopic string one can show that they can all be analytically continued through the horizon. In those cases for which $p$ is even the extension through the horizon is very similar to that described for the eleven-dimensional membrane $(p=2)$ in section 3 ; in each case there is a singularity in the interior region at $r=0$. For $p$ odd, however, the extension is quite different. As will be shown elsewhere [10], the interior region is isometric to the exterior one when $p$ is odd and so there is no singularity there! Thus, unlike the $D=11$ membrane, the $D=10$ three-brane and $D=11$ fivebrane are completely non-singular, despite the presence of an event horizon. Similar remarks apply to the self-dual string [7] in $D=6$.

\section{References}

[1] M.J. Duff and X. Lu, Nucl. Phys. B354 (1991) 141;

[2] G.W. Gibbons and P.K. Townsend, Phys. Rev. Lett. 71 (1993) 3754.

[3] A. Dabholkar, G.W. Gibbons, J.A. Harvey and F. Ruiz-Ruiz, Nucl. Phys. B340 (1990) 33.

[4] M.J. Duff, R.R. Khuri and J.X. Lu, Nucl. Phys. B377 (1992) 281.

[5] M.J. Duff and K.S. Stelle, Phys. Lett. B253 (1991) 113.

[6] M.J. Duff, P.K. Townsend and P. van Nieuwenhuizen, Phys. Lett. 122B (1983) 232.

[7] M.J. Duff and X. Lu, Nucl. Phys. B411 (1994) 301.

[8] G. Horowitz and A. Strominger, Nucl. Phys. B360 (1991) 197.

[9] R. Güven, Phys. Lett. B276 (1992) 49.

[10] G.W. Gibbons, G. Horowitz and P.K. Townsend, in preparation.

* If $d=1$ the potential $A$ is a one-form and there arises the possibility of a Kaluza-Klein interpretation of it as an off-diagonal component of the $(D+1)$-metric. This allows other values of $D$, e.g. $D=4$, but these cases are rather dissimilar to the $D=10$ macroscopic superstring and will not be discussed here. 